PROCEEDINGS OF THE

AMERICAN MATHEMATICAL SOCIETY

Volume 130, Number 4, Pages 1055-1064

S 0002-9939(01)06163-9

Article electronically published on September 14, 2001

\title{
REAL ANALYTIC SOLUTIONS OF PARABOLIC EQUATIONS WITH TIME-MEASURABLE COEFFICIENTS
}

\author{
JAY KOVATS
}

(Communicated by David S. Tartakoff)

\begin{abstract}
We use Bernstein's technique to show that for any fixed $t$, strong solutions $u(t, x)$ of the uniformly parabolic equation $L u:=a^{i j}(t) u_{x_{i} x_{j}}-u_{t}=0$ in $Q$ are real analytic in $Q(t)=\{x:(t, x) \in Q\}$. Here, $Q \subset \mathbb{R}^{d+1}$ is a bounded domain and the coefficients $a^{i j}(t)$ are measurable. We also use Bernstein's technique to obtain interior estimates for pure second derivatives of solutions of the fully nonlinear, uniformly parabolic, concave equation $F\left(D^{2} u, t\right)-u_{t}=0$ in $Q$, where $F$ is measurable in $t$.
\end{abstract}

\section{INTRODUCTION}

In $[\mathrm{B}$, Bernstein introduced a method for estimating the maxima of the moduli of derivatives of any order of solutions of linear parabolic equations, under the assumption that the solution and all known functions of the equation are sufficiently smooth. This technique is outlined in $\S 3.11 .4$ of [La], $\S 4.17$ of [LSU], $\S \S 5.2-5.4$ of [K1], and $\S 8.4$ of [K2]. In the simple linear setting $L u:=a^{i j}(t) u_{x_{i} x_{j}}-u_{t}=0$, the equation can differentiated any number of times in $x$ and so any derivative (in $x$ ) of a solution is again a solution. Note that any difference quotient of any order (in $x$ ) of a solution is also a solution. We assume that the equation is uniformly parabolic; i.e., there exist constants $0<\lambda \leq \Lambda$ with $\lambda|\xi|^{2} \leq a^{i j}(t) \xi_{i} \xi_{j} \leq \Lambda|\xi|^{2}, \forall t$ and $\forall \xi \in \mathbb{R}^{d}$. We assume only that the $a^{i j}(t)$ are measurable functions of $t$. By applying Bernstein's technique, we show that any strong solution of $L u=0$ in $Q$ is real analytic in $x$. This is a generalization of a result which appears in [M], namely if $u(t, x)$ satisfies $\Delta u-u_{t}=0$ in $Q$, then for any fixed $t$, the mapping $x \mapsto u(t, x)$ is analytic. From our result and estimate (1.1) below, it follows that if $u$ is a solution of $L u=0$ in $Q$, then for any fixed $t$, any second order derivative $D^{2} u(t, \cdot)$ is locally Lipschitz continuous in $x$ (see Corollary 1.4).

In $[\mathrm{B}$, Brandt uses only the maximum principle applied to finite difference quotients of solutions to prove that second derivatives of strong solutions of $L u:=$ $a^{i j}(t) u_{x_{i} x_{j}}-u_{t}=f$, where $f(t, x)$ is Hölder continuous in $x$ (with exponent $\alpha \in(0,1))$ are themselves locally Hölder continuous in $x$. In [L], Lieberman uses a Campanato space argument to show that second derivatives are actually locally Hölder continuous in both $t$ and $x$. When $f \equiv 0$, the local Hölder continuity of $D^{2} u$ in $(t, x)$ is an immediate consequence of applying the Hölder continuity of solutions (see [KS] and $\S 4.2$ of [K1]) to solutions $D^{2} u$. Again, these results imply that strong

Received by the editors October 4, 2000.

1991 Mathematics Subject Classification. Primary 35B65, 35K10.

(C)2001 American Mathematical Society 
solutions of $L u=0$ in $Q$ satisfy a $C_{\text {loc }}^{2, \alpha}(Q)$ estimate, where $\alpha \in(0,1)$, while our result implies strong solutions satisfy a $C_{\mathrm{loc}}^{2,1}(Q(t))$ estimate, for any fixed $t$.

The fully nonlinear equation $F\left(D^{2} u, t\right)-u_{t}=0$ is uniformly parabolic if there exist constants $0<\lambda \leq \Lambda$ such that

$$
\lambda\|N\| \leq F(M+N, t)-F(M, t) \leq \Lambda\|N\|,
$$

$\forall M, N \in \mathbb{R}^{d^{2}}$ with $N \geq 0$, where for $B \in \mathbb{R}^{d^{2}},\|B\|=\sqrt{\operatorname{tr}\left(B B^{t}\right)}=\left(\sum_{i, j} b_{i j}^{2}\right)^{1 / 2}$. Under the assumption that $F(M, t)$ is smooth and concave in $M \in \mathbb{R}^{d^{2}}$ and measurable in $t$, we can apply Bernstein's technique to obtain interior estimates on pure second derivatives of solutions of the equation $F\left(D^{2} u, t\right)-u_{t}=0$. This is possible, since under these assumptions, any derivative of a smooth solution will be a solution to a linear parabolic equation with measurable coefficients, while any pure second derivative will be a subsolution of the same linear equation. This was done by Krylov in [K1], [K3] for more general parabolic equations $F\left(D^{2} u, D u, u, t, x\right)-u_{t}=$ 0 , using (in [K1]) a variation of Bernstein's technique called the monitored maximum method. This estimate was the key step towards proving the $W_{2}^{1,2}$ solvability of fully nonlinear equations when $F$ is only measurable in $t$. (See $\S \S 5.4,6.5$ of [K1], $\S 4$ of [K3].)

Our notation is standard. (See, for example, $[\mathrm{GT}], \mathrm{K} 2$.) We remind the reader that for a point $z_{0}=\left(t_{0}, x_{0}\right) \in \mathbb{R}^{d+1}, Q_{\rho}\left(z_{0}\right)=\left(t_{0}-\rho^{2}, t_{0}\right) \times B_{\rho}\left(x_{0}\right)$, while for a function $v,|v|_{0 ; Q}:=\sup _{Q}|v|$. We adopt the convention that repeated indices indicates summation from 1 to $d$, so that, for example, $a^{i j} b^{j i}=\sum_{i, j=1}^{d} a^{i j} b^{j i}=$ $\operatorname{tr}(A B)$.

\section{Linear EqUATIONS With TIME-MEASURABle COEFFICIENTS}

Theorem 1.1. $\forall Q_{\rho}\left(z_{0}\right) \subset \subset Q$ and any multi-index $\alpha$, any $C^{\infty}$ (in $x$ ) solution $u$ of $L u:=a^{i j}(t) u_{x_{i} x_{j}}-u_{t}=0$ in $Q$ satisfies

$$
\left|D^{\alpha} u\left(z_{0}\right)\right| \leq\left(\frac{N|\alpha|}{\rho}\right)^{|\alpha|}|u|_{0 ; Q_{\rho}\left(z_{0}\right)}, \quad \text { where } N=N(d, \lambda, \Lambda) .
$$

Proof. We apply Bernstein's technique. Take any $z_{0}=\left(t_{0}, x_{0}\right) \in Q$ and $\rho>0$ such that $Q_{\rho}\left(z_{0}\right) \subset Q$ and take a function $\varphi \in C^{2}\left(Q_{\rho}\left(z_{0}\right)\right)$ with $0 \leq \varphi \leq 1, \varphi \equiv 0$ on $\partial^{\prime} Q_{\rho}\left(z_{0}\right), \varphi\left(z_{0}\right)=1$ and

$$
|D \varphi|^{2} \leq \frac{N \varphi}{\rho^{2}}, \quad\left\|D^{2} \varphi\right\| \leq \frac{N}{\rho^{2}}, \quad\left|\varphi_{t}\right| \leq \frac{N}{\rho^{2}}
$$

where $N=N(d)$. Consider, in $Q_{\rho}\left(z_{0}\right)$, the function $w=\varphi^{2}|D u|^{2}+C u^{2}$, where the constant $C$ will be determined later. By standard approximation techniques, we may assume $a^{i j}(t) \in C^{\infty}$. Our estimate (1.1) is independent of this smoothness. Then in $Q_{\rho}\left(z_{0}\right)$, since $u$ and each $u_{x_{i}}$ are solutions

$$
\begin{aligned}
L w= & 2|D u|^{2} a^{k j}(t) \varphi_{x_{j}} \varphi_{x_{k}}+2 \varphi|D u|^{2}\left(a^{k j}(t) \varphi_{x_{j} x_{k}}-\varphi_{t}\right) \\
& +8 \varphi a^{k j}(t) \varphi_{x_{j}} \sum_{i} u_{x_{i}} u_{x_{i} x_{k}}+2 \varphi^{2} a^{k j}(t) \sum_{i} u_{x_{i} x_{k}} u_{x_{i} x_{j}} \\
& +2 \varphi^{2} \sum_{i} u_{x_{i}}\left[a^{k j}(t) u_{x_{i} x_{j} x_{k}}-u_{x_{i} t}\right]+2 C a^{k j}(t) u_{x_{k}} u_{x_{j}}
\end{aligned}
$$




$$
\begin{aligned}
& +2 C u\left(a^{k j}(t) u_{x_{j} x_{k}}-u_{t}\right) \\
= & 2|D u|^{2} a^{k j}(t) \varphi_{x_{j}} \varphi_{x_{k}}+2 \varphi|D u|^{2}\left(a^{k j}(t) \varphi_{x_{j} x_{k}}-\varphi_{t}\right) \\
& +8 \varphi a^{k j}(t) \varphi_{x_{j}} \sum_{i} u_{x_{i}} u_{x_{i} x_{k}}+2 \varphi^{2} a^{k j}(t) \sum_{i} u_{x_{i} x_{k}} u_{x_{i} x_{j}}+2 C a^{k j}(t) u_{x_{k}} u_{x_{j}} \\
\geq & 2|D u|^{2} \lambda|D \varphi|^{2}+2 \varphi|D u|^{2}\left(a^{k j}(t) \varphi_{x_{j} x_{k}}-\varphi_{t}\right) \\
& -8 \varphi \Lambda|D \varphi| \cdot|D u| \cdot\left\|D^{2} u\right\|+2 \varphi^{2} \lambda\left\|D^{2} u\right\|^{2}+2 C \lambda|D u|^{2} \\
\geq & 2|D u|^{2} \lambda|D \varphi|^{2}+2 \varphi|D u|^{2}\left(a^{k j}(t) \varphi_{x_{j} x_{k}}-\varphi_{t}+C \lambda\right) \\
& -8 \varphi \Lambda|D \varphi| \cdot|D u| \cdot\left\|D^{2} u\right\|+2 \varphi^{2} \lambda\left\|D^{2} u\right\|^{2}
\end{aligned}
$$

since $0 \leq \varphi \leq 1$ and $\sum_{i} a^{k j}(t) u_{x_{i} x_{k}} u_{x_{i} x_{j}} \geq \sum_{i} \lambda\left|D\left(u_{x_{i}}\right)\right|^{2}=\lambda \sum_{i, k} u_{x_{i} x_{k}}^{2}=\lambda\left\|D^{2} u\right\|^{2}$. In the second to the last inequality we have used Cauchy's inequality and the fact that $\left|a^{k j} x_{k} y_{j}\right| \leq \sqrt{a^{k j} x_{k} x_{j}} \sqrt{a^{k j} y_{k} y_{j}}$ for any $x, y \in \mathbb{R}^{d}$. First choose $C$ so large that $\left|a^{i j}(t) \varphi_{x_{i} x_{j}}-\varphi_{t}\right| \leq \frac{C \lambda}{2}$. Observe that $\left|a^{i j} \varphi_{x_{i} x_{j}}-\varphi_{t}\right| \leq\|A\| \cdot\left\|D^{2} \varphi\right\|+\left|\varphi_{t}\right| \leq$ $\frac{\Lambda d^{1 / 2} N}{\rho^{2}}+\frac{N}{\rho^{2}}=\frac{N}{\rho^{2}}\left(\Lambda d^{1 / 2}+1\right)$. So $C \geq \frac{2 N}{\lambda \rho^{2}}\left(\Lambda d^{1 / 2}+1\right)$ will do. By Young's inequality $\forall \varepsilon>0$ we have

$$
\begin{aligned}
L w \geq & 2|D u|^{2} \lambda|D \varphi|^{2}+\varphi|D u|^{2} C \lambda-8 \Lambda\left(\varepsilon|D u|^{2}|D \varphi|^{2}+\frac{\varphi^{2}\left\|D^{2} u\right\|^{2}}{\varepsilon}\right) \\
& +2 \varphi^{2} \lambda\left\|D^{2} u\right\|^{2} \\
\geq & |D u|^{2}|D \varphi|^{2}\left(2 \lambda-8 \Lambda \varepsilon+\frac{C \lambda \rho^{2}}{N}\right)+\varphi^{2}\left\|D^{2} u\right\|^{2}\left(2 \lambda-\frac{8 \Lambda}{\varepsilon}\right) \\
\geq & |D u|^{2}|D \varphi|^{2}\left(\frac{C \lambda \rho^{2}}{N}-8 \Lambda \varepsilon\right)+\varphi^{2}\left\|D^{2} u\right\|^{2}\left(2 \lambda-\frac{8 \Lambda}{\varepsilon}\right)
\end{aligned}
$$

Now choose $\varepsilon$ st $\frac{C \lambda \rho^{2}}{N}-8 \Lambda \varepsilon=\frac{C \lambda \rho^{2}}{2 N}$. This gives

$$
L w \geq|D u|^{2}|D \varphi|^{2} \frac{C \lambda \rho^{2}}{2 N}+\varphi^{2}\left\|D^{2} u\right\|^{2}\left(2 \lambda-\frac{128 \Lambda^{2} N}{C \lambda \rho^{2}}\right) .
$$

Finally, choose $C$ so large that $2 \lambda-\frac{128 \Lambda^{2} N}{C \lambda \rho^{2}} \geq 0$. Observe that for

$$
C=\max \left\{\frac{2 N}{\lambda \rho^{2}}\left(\Lambda d^{1 / 2}+1\right), \frac{64 \Lambda^{2} N}{\lambda^{2} \rho^{2}}\right\},
$$

we have $L w \geq 0$ in $Q_{\rho}\left(z_{0}\right)$. Hence by the maximum principle $\sup _{Q_{\rho}\left(z_{0}\right)} w=\sup _{\partial^{\prime} Q_{\rho}\left(z_{0}\right)} w$ and so

$$
\left|D u\left(z_{0}\right)\right|^{2} \leq C \sup _{Q_{\rho}\left(z_{0}\right)} u^{2}=\frac{N_{1}(\lambda, \Lambda, d)}{\rho^{2}} \sup _{Q_{\rho}\left(z_{0}\right)} u^{2},
$$

which gives (1.1) for $|\alpha|=1$. So assume our claim is true for $|\alpha|=k$. We verify it holds for $|\alpha|=k+1$. Fix $z_{0} \in Q$ and $\rho>0$ such that $Q_{\rho}\left(z_{0}\right) \subset Q$. Then $Q_{\frac{\rho}{k+1}}\left(z_{0}\right) \subset Q_{\rho}\left(z_{0}\right) \subset Q$ and $\alpha=\beta+e_{i}$, for some $i=1, \ldots, d$, where $|\beta|=k$. Moreover for $z \in Q_{\frac{\rho}{k+1}}\left(z_{0}\right)$, we have $Q_{\frac{k \rho}{k+1}}(z) \subset Q_{\rho}\left(z_{0}\right)$ and hence by the inductive 
hypothesis

$$
\begin{aligned}
\left|D^{\alpha} u\left(z_{0}\right)\right| & =\left|\left(D^{\beta} u\left(z_{0}\right)\right)_{x_{i}}\right| \leq \frac{N}{\left(\frac{\rho}{k+1}\right)}\left|D^{\beta} u\right|_{0 ; Q_{\frac{\rho}{k+1}}}\left(z_{0}\right) \\
& \leq \frac{N}{\left(\frac{\rho}{k+1}\right)} \cdot\left(\frac{N k}{\left(\frac{k \rho}{k+1}\right)}\right)^{k}|u|_{0 ; Q_{\rho}\left(z_{0}\right)} \\
& =\left(\frac{N(k+1)}{\rho}\right)^{k+1}|u|_{0 ; Q_{\rho}\left(z_{0}\right)} .
\end{aligned}
$$

Lemma 1.2. Any strong solution $u=u(t, x)$ of $L u:=a^{i j}(t) u_{x_{i} x_{j}}-u_{t}=0$ in $Q$ is $C^{\infty}$ in $x$.

Proof. For our solution $u$, consider $u^{\epsilon}$, the mollifier (in $x$ ) of $u$. For any fixed $t$ and any $x$

$$
\begin{aligned}
\left(u^{\epsilon}\right)_{t}(t, x) & =\left(u_{t}\right)^{\epsilon}(t, x)=\left(a^{i j}(t) u_{x_{i} x_{j}}\right)^{\epsilon}(t, x) \\
& =a^{i j}(t)\left(u_{x_{i} x_{j}}\right)^{\epsilon}(t, x)=a^{i j}(t)\left(u^{\epsilon}\right)_{x_{i} x_{j}}(t, x),
\end{aligned}
$$

and hence $u^{\epsilon}$ is a $C^{\infty}$ (in $x$ ) solution of $L u^{\epsilon}=0$ and hence by Theorem 1, for any $\alpha$

$$
\left|D^{\alpha} u^{\epsilon}\left(z_{0}\right)\right| \leq\left(\frac{N|\alpha|}{\rho}\right)^{|\alpha|}\left|u^{\epsilon}\right|_{0 ; Q_{\rho}\left(z_{0}\right)} \leq\left(\frac{N|\alpha|}{\rho}\right)^{|\alpha|}|u|_{0 ; Q} .
$$

That is, for any $\alpha$, the sequence $\left\{D^{\alpha} u^{\epsilon}\right\}$ is uniformly bounded and equicontinuous. By the Arzela-Ascoli theorem, it has a subsequence $\left\{D^{\alpha} u^{\epsilon}\right\}$ (by relabelling if necessary) converging uniformly on compacta of $Q$ to some continuous function $v=v_{\alpha}$. Hence $\forall \xi \in C_{0}^{|\alpha|}(Q(t))$, where $Q(t)=\{x:(t, x) \in Q\}$, integration by parts gives

$$
\begin{gathered}
(-1)^{|\alpha|} \int_{Q(t)} u(t, x) D^{\alpha} \xi(x) d x=\lim _{\epsilon \rightarrow 0}(-1)^{|\alpha|} \int_{Q(t)} u^{\epsilon}(t, x) D^{\alpha} \xi(x) d x \\
=\lim _{\epsilon \rightarrow 0} \int_{Q(t)} D^{\alpha}\left(u^{\epsilon}\right)(t, x) \xi(x) d x=\int_{Q(t)} v(t, x) \xi(x) d x
\end{gathered}
$$

that is, $D^{\alpha} u=v$ in the weak sense. But (1.2) and the fact that $\left\{D^{\alpha} u^{\epsilon}\right\} \rightarrow v$ implies $v \in L_{\mathrm{loc}}^{\infty}(Q)$, i.e. $u(t, \cdot) \in W_{\mathrm{loc}}^{|\alpha|, \infty}(Q(t))=C^{|\alpha|-1,1}(Q(t))$. Since $\alpha$ is arbitrary, $u(t, \cdot) \in C^{\infty}(Q(t))$.

Theorem 1.3. If $Q \subset \mathbb{R}^{d+1}$ is a bounded domain, any solution $u(t, x)$ of $L u:=$ $a^{i j}(t) u_{x_{i} x_{j}}-u_{t}=0$ in $Q$ is real analytic in $x$.

Proof. By Lemma 1.2, we know $u$ is $C^{\infty}$ in $x$. Fix any point $z_{0}=\left(t_{0}, x_{0}\right) \in Q$. We will show that $u$ can be represented as a power series (in $x$ ) in a neighborhood of $z_{0}$. So set $\rho=\frac{1}{4} \operatorname{dist}\left(z_{0}, \partial^{\prime} Q\right)$. Observe that for $z \in Q_{\rho}\left(z_{0}\right), Q_{\rho}(z) \subset Q_{2 \rho}\left(z_{0}\right) \subset Q$ and hence for any such $z$, by Theorem 1 we have $\left|D^{\alpha} u(z)\right| \leq\left(\frac{N|\alpha|}{\rho}\right)^{|\alpha|}|u|_{0 ; Q_{\rho}(z)} \leq$ 
$\left(\frac{N|\alpha|}{\rho}\right)^{|\alpha|}|u|_{0 ; Q_{2 \rho}\left(z_{0}\right)}:=\left(\frac{N|\alpha|}{\rho}\right)^{|\alpha|} M$. By Taylor's theorem, for any $k \geq 1$ we have

$$
u(t, x)=\sum_{|\alpha| \leq k-1} \frac{D^{\alpha} u\left(t_{0}, x_{0}\right)}{\alpha !}\left(x-x_{0}\right)^{\alpha}+\sum_{|\alpha|=k} \frac{D^{\alpha} u\left(t_{0}, \xi\right)}{\alpha !}\left(x-x_{0}\right)^{\alpha},
$$

where $\xi=\gamma x+(1-\gamma) x_{0}$ for some $\gamma \in(0,1)$. Since $\left(t_{0}, \xi\right) \in Q_{\rho}\left(z_{0}\right)$, we have $\left|D^{\alpha} u\left(t_{0}, \xi\right)\right| \leq\left(\frac{N|\alpha|}{\rho}\right)^{|\alpha|} M$. Observe that the second sum on the right goes to zero as $k \rightarrow \infty$ for $\left|x-x_{0}\right| \leq \frac{\rho}{2 N d e}$, since by a strong version of Stirling's formula, $k^{k}<\frac{e^{k} k !}{\sqrt{2 \pi}}$, we have

$$
\begin{aligned}
\sum_{|\alpha|=k} \frac{\left|D^{\alpha} u\left(t_{0}, \xi\right)\right|}{\alpha !}\left|x-x_{0}\right|^{|\alpha|} & \leq \sum_{|\alpha|=k} \frac{1}{\alpha !}\left(\frac{N|\alpha|}{\rho}\right)^{|\alpha|} M\left(\frac{\rho}{2 N d e}\right)^{|\alpha|} \\
& =\frac{M}{(2 d e)^{k}} \sum_{|\alpha|=k} \frac{|\alpha|^{|\alpha|}}{\alpha !} \\
& <\frac{M}{(2 d e)^{k}} \sum_{|\alpha|=k} \frac{|\alpha| ! e^{|\alpha|}}{\sqrt{2 \pi} \alpha !} \\
& =\frac{M}{(2 d)^{k} \sqrt{2 \pi}} \sum_{|\alpha|=k} \frac{|\alpha| !}{\alpha !} \\
& =\frac{M}{(2 d)^{k} \sqrt{2 \pi}} \cdot d^{k} \rightarrow 0 \text { as } k \rightarrow \infty,
\end{aligned}
$$

and so $u(t, x)=\sum_{\alpha} \frac{D^{\alpha} u\left(t_{0}, x_{0}\right)}{\alpha !}\left(x-x_{0}\right)^{\alpha}$.

Corollary 1.4. If $Q \subset \mathbb{R}^{d+1}$ is a bounded domain, any solution $u(t, x)$ of $L u:=$ $a^{i j}(t) u_{x_{i} x_{j}}-u_{t}=0$ in $Q$ has locally Lipschitz second derivatives (in $x$ ). That is, for any fixed $t, u(t, \cdot) \in C_{\text {loc }}^{2,1}(Q(t))$ and for any $(t, x),(t, y) \in Q$ and $i, j=1, \ldots, d$, we have

$$
\left|u_{x_{i} x_{j}}(t, x)-u_{x_{i} x_{j}}(t, y)\right| \leq \frac{N_{1}}{\rho^{3}} \cdot|u|_{0 ; Q} \cdot|x-y|,
$$

where $N_{1}=N_{1}(d, \lambda, \Lambda)$ and $\rho=\frac{1}{4} \min \left\{d_{z_{x}}, d_{z_{y}}\right\}$, where $d_{z_{x}}=\operatorname{dist}\left((t, x), \partial^{\prime} Q\right)$.

Proof. Fix $(t, x),(t, y) \in Q$. Denote these points $z_{x}, z_{y}$, respectively and set $\rho=$ $\frac{1}{4} \min \left\{d_{z_{x}}, d_{z_{y}}\right\}$. Then either $z_{y} \in Q_{\rho}\left(z_{x}\right)$ or $z_{y} \notin Q_{\rho}\left(z_{x}\right)$. If $z_{y} \in Q_{\rho}\left(z_{x}\right)$, then $z_{\xi}=(t, \xi) \in Q_{\rho}\left(z_{x}\right)$, where $\xi=\gamma x+(1-\gamma) y$ and $\gamma \in(0,1)$. Hence $Q_{\rho}\left(z_{\xi}\right) \subset$ $Q_{2 \rho}\left(z_{x}\right) \subset Q$ and so by the mean-value theorem and Theorem 1.1, we get

$$
\begin{aligned}
& \left|u_{x_{i} x_{j}}(t, x)-u_{x_{i} x_{j}}(t, y)\right| \leq\left|D\left(u_{x_{i} x_{j}}\right)(t, \xi)\right| \cdot|x-y| \\
& \quad \leq\left(\frac{N 3}{\rho}\right)^{3}|u|_{0 ; Q_{\rho}\left(z_{\xi}\right)} \cdot|x-y| \leq\left(\frac{N 3}{\rho}\right)^{3}|u|_{0 ; Q} \cdot|x-y| .
\end{aligned}
$$


On the other hand, if $z_{y} \notin Q_{\rho}\left(z_{x}\right)$, i.e., $|x-y| \geq \rho$, then since $z_{x} \in Q_{d_{z_{x}}}\left(z_{x}\right) \subset Q$, Theorem 1.1 gives

$$
\begin{aligned}
\frac{\left|u_{x_{i} x_{j}}(t, x)-u_{x_{i} x_{j}}(t, y)\right|}{|x-y|} & \leq \frac{\left|u_{x_{i} x_{j}}(t, x)\right|+\left|u_{x_{i} x_{j}}(t, y)\right|}{\rho} \\
& \leq \frac{1}{\rho}\left[\left(\frac{N 2}{d_{z_{x}}}\right)^{2}|u|_{0 ; Q_{d_{z_{x}}}\left(z_{x}\right)}+\left(\frac{N 2}{d_{z_{y}}}\right)^{2}|u|_{0 ; Q_{d_{z_{y}}}}\left(z_{y}\right)\right] \\
& \leq \frac{1}{\rho}\left[\left(\frac{N 2}{4 \rho}\right)^{2}|u|_{0 ; Q}+\left(\frac{N 2}{4 \rho}\right)^{2}|u|_{0 ; Q}\right]=\frac{N^{2}}{2 \rho^{3}}|u|_{0 ; Q}
\end{aligned}
$$

so in either case we get (1.3).

\section{Simple FUlly NONLinear EQUATIONS WITH TIME-MEASURABLE COEFFICIENTS}

In what follows we assume that the nonlinear operator $F(M, t)$ is smooth and concave in $M \in \mathbb{R}^{d^{2}}$ and measurable in $t$. Since $F$ is differentiable in $M$, our uniform parabolicity condition (0.1) now reads

$$
\lambda|\xi|^{2} \leq F_{i j}(M, t) \xi_{i} \xi_{j} \leq \Lambda|\xi|^{2}, \quad \text { where } F_{i j}=\frac{\partial F}{\partial m_{i j}},
$$

while the concavity of $F$ in $M$ implies $N^{t} \cdot D^{2} F(M, t) \cdot N \leq 0, \forall N \in \mathbb{R}^{d^{2}}$, i.e. $F_{i j, k l}(M, t) N_{i j} N_{k l} \leq 0$, where $F_{i j, k l}=\frac{\partial^{2} F}{\partial m_{i j} \partial m_{k l}}$.

Lemma 2.1. Let $u$ be a smooth solution of the equation $F\left(D^{2} u, t\right)-u_{t}=0$ in $Q$, where $F(0, \cdot)=0$. Then there exists a linear operator $L:=a^{i j}(t, x) \frac{\partial^{2}}{\partial x_{i} \partial x_{j}}-\frac{\partial}{\partial t}$, with $\lambda|\xi|^{2} \leq a^{i j}(t, x) \xi_{i} \xi_{j} \leq \Lambda|\xi|^{2}$, such that $L u \leq 0$ and $\forall|e|=1, L u_{e}=0$ and $L u_{e e} \geq 0$ in $Q$.

Proof. Differentiating the equation $u_{t}(t, x)=F\left(D^{2} u(t, x), t\right)$ with respect to any vector $|e|=1$ gives

$$
\left(u_{e}\right)_{t}(t, x)=F_{i j}\left(D^{2} u(t, x), t\right)\left(u_{e}\right)_{x_{i} x_{j}}:=a^{i j}(t, x)\left(u_{e}\right)_{x_{i} x_{j}} .
$$

Thus $L u_{e}=0$ in $Q$, where $a^{i j}(t, x)$ satisfies (2.0). Differentiating the equation again with respect to $e$ (omitting the obvious argument $\left(D^{2} u(t, x), t\right)$ ) and using the fact that $F$ is concave gives

$$
\left(u_{e e}\right)_{t}(t, x)=F_{i j, k l}\left(u_{e}\right)_{x_{k} x_{l}}\left(u_{e}\right)_{x_{i} x_{j}}+F_{i j}\left(u_{e e}\right)_{x_{i} x_{j}} \leq F_{i j}\left(u_{e e}\right)_{x_{i} x_{j}},
$$

which gives $L u_{e e} \geq 0$ in $Q$. Finally, to conclude $L u \leq 0$, observe that since $F$ is concave, so is $g(r)=F\left((1-r) D^{2} u(t, x), t\right)$. Hence $\forall r$ we have $g(r) \leq g^{\prime}(0) r+g(0)$. In particular for $r=1, g(1) \leq g^{\prime}(0)+g(0)$, which from the equation and $F(0, t)=0$ gives $0 \leq-a^{i j}(t, x) u_{x_{i} x_{j}}+u_{t}$.

For fixed $\rho>0$, we define $Q^{\rho}:=\left\{z_{0} \in Q: Q_{\rho}\left(z_{0}\right) \subset Q\right\}$. We now prove the interior estimates for pure second derivatives of solutions of $F\left(D^{2} u, t\right)-u_{t}=0$ in $Q$, where $F(0, \cdot)=0$. For a proof of this for fully nonlinear constant coefficient elliptic equations, see $\S 9.1$ of [CC]. In $\S \S 5.2-5.4$ of [K1], as well as $\S 4$ in [K3], Krylov proves this result for more general parabolic equations $F\left(D^{2} u, D u, u, t, x\right)-u_{t}=0$, 
under additional assumptions on $F$, using a variation of Bernstein's technique he calls the monitored maximum method. This interior estimate for $u_{e e}$ led to interior estimates for $\left\|u_{t}\right\|_{L^{2}},\left\|D^{2} u\right\|_{L^{2}}$, which in turn led to the $W_{2}^{1,2}$ solvability of fully nonlinear equations when $F$ is only measurable in $t$. The proof of this was based on the $C^{2, \alpha}$ solvability for functions $F$ which are smooth in $t$. (See $\S \S 5.4,6.5$ of [K1.)

Theorem 2.2. Let $u$ be a $C^{4}$ solution of the concave equation $F\left(D^{2} u, t\right)-u_{t}=0$ in $Q$, where $F \in C^{2}\left(\mathbb{R}^{d^{2}}\right)$ and $F(0, \cdot)=0$. Then there is a constant $N=N(d, \lambda, \Lambda)$ such that $\forall e \in \mathbb{R}^{d}$ with $|e|=1$

$$
\left|u_{e e}^{+}\right|_{0 ; Q^{\rho}} \leq \frac{N}{\rho^{2}}|u|_{0 ; Q}
$$

Proof. For fixed $\rho>0$, recall $Q^{\rho}:=\left\{z_{0} \in Q: Q_{\rho}\left(z_{0}\right) \subset Q\right\}$ So take any $z_{0}=$ $\left(t_{0}, x_{0}\right) \in Q^{\rho}$ and take a function $\varphi \in C^{2}\left(Q_{\rho}\left(z_{0}\right)\right)$ with $0 \leq \varphi \leq 1, \varphi \equiv 0$ on $\partial^{\prime} Q_{\rho}\left(z_{0}\right), \varphi\left(z_{0}\right)=1$ and

$$
|D \varphi|^{2} \leq \frac{N \varphi}{\rho^{2}}, \quad\left\|D^{2} \varphi\right\| \leq \frac{N}{\rho^{2}}, \quad\left|\varphi_{t}\right| \leq \frac{N}{\rho^{2}}
$$

where $N=N(d)$. As in Theorem 1.1, for a constant $C$ to be determined later, consider, in $Q_{\rho}\left(z_{0}\right)$, the function

$$
v=\varphi^{2}\left(u_{e e}^{+}\right)^{2}+C u_{e}^{2}
$$

In $Q_{\rho}\left(z_{0}\right) \cap\left\{u_{e e} \geq 0\right\}$, for $L:=a^{i j}(t, x) \frac{\partial^{2}}{\partial x_{i} \partial x_{j}}-\frac{\partial}{\partial t}$, we have, since $L u_{e}=0, L u_{e e} \geq 0$ by Lemma 2.1

$$
\begin{aligned}
L v= & 2 u_{e e}^{2} a^{i j} \varphi_{x_{i}} \varphi_{x_{j}}+2 \varphi u_{e e}^{2}\left(a^{i j} \varphi_{x_{i} x_{j}}-\varphi_{t}\right)+8 \varphi u_{e e} a^{i j} \varphi_{x_{i}} u_{e e x_{j}} \\
& +2 \varphi^{2} a^{i j} u_{e e x_{j}} u_{e e x_{i}}+2 \varphi^{2} u_{e e}\left(a^{i j} u_{e e x_{i} x_{j}}-u_{e e t}\right) \\
& +2 C a^{i j} u_{e x_{j}} u_{e x_{i}}+2 C u_{e}\left(a^{i j} u_{e x_{i} x_{j}}-u_{e t}\right) \\
\geq & 2 u_{e e}^{2} a^{i j} \varphi_{x_{i}} \varphi_{x_{j}}+2 \varphi u_{e e}^{2}\left(a^{i j} \varphi_{x_{i} x_{j}}-\varphi_{t}\right)+8 \varphi u_{e e} a^{i j} \varphi_{x_{i}} u_{e e x_{j}} \\
& +2 \varphi^{2} a^{i j} u_{e e x_{j}} u_{e e x_{i}}+2 C a^{i j} u_{e x_{j}} u_{e x_{i}} \\
\geq & 2 u_{e e}^{2} \lambda|D \varphi|^{2}+2 \varphi u_{e e}^{2}\left(a^{i j} \varphi_{x_{i} x_{j}}-\varphi_{t}\right)-8 \Lambda \varphi u_{e e}|D \varphi| \cdot\left|D u_{e e}\right| \\
& +2 \varphi^{2} \lambda\left|D u_{e e}\right|^{2}+2 C \lambda\left|D u_{e}\right|^{2} \\
\geq & 2 u_{e e}^{2} \lambda|D \varphi|^{2}+2 \varphi u_{e e}^{2}\left(a^{i j} \varphi_{x_{i} x_{j}}-\varphi_{t}+C \lambda\right)-8 \Lambda \varphi u_{e e}|D \varphi| \cdot\left|D u_{e e}\right| \\
& +2 \varphi^{2} \lambda\left|D u_{e e}\right|^{2},
\end{aligned}
$$

since $0 \leq \varphi \leq 1$ and $u_{e e}=D u_{e} \cdot e \leq\left|D u_{e}\right|$. First choose $C$ so large that $\mid a^{i j} \varphi_{x_{i} x_{j}}-$ $\varphi_{t} \mid \leq \frac{C \lambda}{2}$. Observe that $\left|a^{i j} \varphi_{x_{i} x_{j}}-\varphi_{t}\right| \leq\|A\| \cdot\left\|D^{2} \varphi\right\|+\left|\varphi_{t}\right| \leq \frac{\Lambda d^{1 / 2} N}{\rho^{2}}+\frac{N}{\rho^{2}}=$ $\frac{N}{\rho^{2}}\left(\Lambda d^{1 / 2}+1\right)$. So $C \geq \frac{2 N}{\lambda \rho^{2}}\left(\Lambda d^{1 / 2}+1\right)$ will do. By Young's inequality $\forall \varepsilon>0$ we 
have

$$
\begin{aligned}
L v & \geq 2 u_{e e}^{2} \lambda|D \varphi|^{2}+\varphi u_{e e}^{2} C \lambda-8 \Lambda\left(\varepsilon u_{e e}^{2}|D \varphi|^{2}+\frac{\varphi^{2}\left|D u_{e e}\right|^{2}}{\varepsilon}\right)+2 \varphi^{2} \lambda\left|D u_{e e}\right|^{2} \\
& =u_{e e}^{2}|D \varphi|^{2}(2 \lambda-8 \Lambda \varepsilon)+\varphi u_{e e}^{2} C \lambda+\varphi^{2}\left|D u_{e e}\right|^{2}\left(2 \lambda-\frac{8 \Lambda}{\varepsilon}\right) \\
& \geq u_{e e}^{2}|D \varphi|^{2}(2 \lambda-8 \Lambda \varepsilon)+\frac{u_{e e}^{2} C \lambda|D \varphi|^{2} \rho^{2}}{N}+\varphi^{2}\left|D u_{e e}\right|^{2}\left(2 \lambda-\frac{8 \Lambda}{\varepsilon}\right) \\
& =u_{e e}^{2}|D \varphi|^{2}\left(2 \lambda-8 \Lambda \varepsilon+\frac{C \lambda \rho^{2}}{N}\right)+\varphi^{2}\left|D u_{e e}\right|^{2}\left(2 \lambda-\frac{8 \Lambda}{\varepsilon}\right) \\
& \geq u_{e e}^{2}|D \varphi|^{2}\left(\frac{C \lambda \rho^{2}}{N}-8 \Lambda \varepsilon\right)+\varphi^{2}\left|D u_{e e}\right|^{2}\left(2 \lambda-\frac{8 \Lambda}{\varepsilon}\right) .
\end{aligned}
$$

Now choose $\varepsilon$ st $\frac{C \lambda \rho^{2}}{N}-8 \Lambda \varepsilon=\frac{C \lambda \rho^{2}}{2 N}$. This gives

$$
L v \geq u_{e e}^{2}|D \varphi|^{2} \frac{C \lambda \rho^{2}}{2 N}+\varphi^{2}\left|D u_{e e}\right|^{2}\left(2 \lambda-\frac{128 \Lambda^{2} N}{C \lambda \rho^{2}}\right) .
$$

Finally, choose $C$ so large that $2 \lambda-\frac{128 \Lambda^{2} N}{C \lambda \rho^{2}} \geq 0$. Observe that for

$$
C=\max \left\{\frac{2 N}{\lambda \rho^{2}}\left(\Lambda d^{1 / 2}+1\right), \frac{64 \Lambda^{2} N}{\lambda^{2} \rho^{2}}\right\},
$$

we have $L v \geq 0$ in $Q_{\rho}\left(z_{0}\right) \cap\left\{u_{e e} \geq 0\right\}$. But in $Q_{\rho}\left(z_{0}\right) \cap\left\{u_{e e} \leq 0\right\}, v=C u_{e}^{2}$ and since $L u_{e}=0$, an easy calculation gives $L v=C L\left(u_{e}^{2}\right) \geq 2 C \lambda\left|D\left(u_{e}\right)\right|^{2} \geq 0$. Thus $L v \geq 0$ in $Q_{\rho}\left(z_{0}\right)$. Hence by the maximum principle $\sup _{Q_{\rho}\left(z_{0}\right)} v=\sup _{\partial^{\prime} Q_{\rho}\left(z_{0}\right)} v$ and so $\left(u_{e e}^{+}\right)^{2}\left(z_{0}\right) \leq C \sup _{Q_{\rho}\left(z_{0}\right)} u_{e}^{2}=\frac{N_{1}(\lambda, \Lambda, d)}{\rho^{2}} \sup _{Q_{\rho}\left(z_{0}\right)} u_{e}^{2} \leq \frac{N_{1}}{\rho^{2}} \sup _{Q} u_{e}^{2}$ and since $z_{0} \in Q^{\rho}$ was arbitrary, we have

$$
\sup _{Q^{\rho}} u_{e e}^{+} \leq \frac{N_{2}}{\rho} \sup _{Q}\left|u_{e}\right|
$$

Finally, to get an estimate for $\left|u_{e}\right|$, we apply Bernstein's technique to the function

$$
w=\varphi^{2} u_{e}^{2}+C(M-u)^{2}
$$

in $Q_{\rho}\left(z_{0}\right)$, where $M:=|u|_{0 ; Q_{\rho}\left(z_{0}\right)}$. By Lemma $2.1, L u_{e}=0, L u \leq 0$ and hence

$$
\begin{aligned}
L w= & 2 u_{e}^{2} a^{i j} \varphi_{x_{i}} \varphi_{x_{j}}+2 \varphi u_{e}^{2}\left(a^{i j} \varphi_{x_{i} x_{j}}-\varphi_{t}\right)+8 \varphi u_{e} a^{i j} \varphi_{x_{i}} u_{e x_{j}}+2 \varphi^{2} a^{i j} u_{e x_{j}} u_{e x_{i}} \\
& +2 \varphi^{2} u_{e}\left[a^{i j} u_{e x_{i} x_{j}}-u_{e t}\right]+2 C a^{i j} u_{x_{i}} u_{x_{j}}-2 C(M-u)\left(a^{i j} u_{x_{i} x_{j}}-u_{t}\right) \\
\geq & 2 u_{e}^{2} a^{i j} \varphi_{x_{i}} \varphi_{x_{j}}+2 \varphi u_{e}^{2}\left(a^{i j} \varphi_{x_{i} x_{j}}-\varphi_{t}\right)+8 \varphi u_{e} a^{i j} \varphi_{x_{i}} u_{e x_{j}} \\
& +2 \varphi^{2} a^{i j} u_{e x_{j}} u_{e x_{i}}+2 C a^{i j} u_{x_{i}} u_{x_{j}} \\
\geq & 2 u_{e}^{2} \lambda|D \varphi|^{2}+2 \varphi u_{e}^{2}\left(a^{i j} \varphi_{x_{i} x_{j}}-\varphi_{t}\right)-8 \varphi \Lambda|D \varphi| \cdot\left|u_{e}\right| \cdot\left|D\left(u_{e}\right)\right| \\
& +2 \varphi^{2} \lambda\left|D\left(u_{e}\right)\right|^{2}+2 C \lambda|D u|^{2} \\
\geq & 2 u_{e}^{2} \lambda|D \varphi|^{2}+2 \varphi u_{e}^{2}\left(a^{i j} \varphi_{x_{i} x_{j}}-\varphi_{t}+C \lambda\right)-8 \varphi \Lambda|D \varphi| \cdot\left|u_{e}\right| \cdot\left|D\left(u_{e}\right)\right| \\
& +2 \varphi^{2} \lambda\left|D\left(u_{e}\right)\right|^{2}
\end{aligned}
$$

since $0 \leq \varphi \leq 1,\left|u_{e}\right| \leq|D u|$ and $\left|a^{i j} \varphi_{x_{i}} u_{e x_{j}}\right| \leq \sqrt{a^{i j} \varphi_{x_{i}} \varphi_{x_{j}}} \sqrt{a^{i j} u_{e x_{i}} u_{e x_{j}}} \leq$ $\Lambda|D \varphi| \cdot\left|D\left(u_{e}\right)\right|$. First choose $C$ so large that $\left|a^{i j} \varphi_{x_{i} x_{j}}-\varphi_{t}\right| \leq \frac{C \lambda}{2}$. By Young's 
inequality $\forall \varepsilon>0$ we have

$$
\begin{aligned}
L w & \geq 2 u_{e}^{2} \lambda|D \varphi|^{2}+\varphi u_{e}^{2} C \lambda-8 \Lambda\left(\varepsilon u_{e}^{2}|D \varphi|^{2}+\frac{\varphi^{2}\left|D\left(u_{e}\right)\right|^{2}}{\varepsilon}\right)+2 \varphi^{2} \lambda\left|D\left(u_{e}\right)\right|^{2} \\
& \geq u_{e}^{2}|D \varphi|^{2}\left(2 \lambda-8 \Lambda \varepsilon+\frac{C \lambda \rho^{2}}{N}\right)+\varphi^{2}\left|D\left(u_{e}\right)\right|^{2}\left(2 \lambda-\frac{8 \Lambda}{\varepsilon}\right) \\
& \geq u_{e}^{2}|D \varphi|^{2}\left(\frac{C \lambda \rho^{2}}{N}-8 \Lambda \varepsilon\right)+\varphi^{2}\left|D\left(u_{e}\right)\right|^{2}\left(2 \lambda-\frac{8 \Lambda}{\varepsilon}\right)
\end{aligned}
$$

Now choose $\varepsilon$ st $\frac{C \lambda \rho^{2}}{N}-8 \Lambda \varepsilon=\frac{C \lambda \rho^{2}}{2 N}$. This gives

$$
L w \geq u_{e}^{2}|D \varphi|^{2} \frac{C \lambda \rho^{2}}{2 N}+\varphi^{2}\left|D\left(u_{e}\right)\right|^{2}\left(2 \lambda-\frac{128 \Lambda^{2} N}{C \lambda \rho^{2}}\right) .
$$

Finally, choose $C$ so large that $2 \lambda-\frac{128 \Lambda^{2} N}{C \lambda \rho^{2}} \geq 0$. Observe that for

$$
C=\max \left\{\frac{2 N}{\lambda \rho^{2}}\left(\Lambda d^{1 / 2}+1\right), \frac{64 \Lambda^{2} N}{\lambda^{2} \rho^{2}}\right\},
$$

we have $L w \geq 0$ in $Q_{\rho}\left(z_{0}\right)$. Hence by the maximum principle $\sup _{Q_{\rho}\left(z_{0}\right)} w=\sup _{\partial^{\prime} Q_{\rho}\left(z_{0}\right)} w$ and so $u_{e}^{2}\left(z_{0}\right) \leq C \sup _{Q_{\rho}\left(z_{0}\right)}(M-u)^{2}=\frac{N_{1}(\lambda, \Lambda, d)}{\rho^{2}} \sup _{Q_{\rho}\left(z_{0}\right)}(M-u)^{2} \leq \frac{4 N_{1}}{\rho^{2}}\left(|u|_{0 ; Q}\right)^{2}$. Since $z_{0} \in Q^{\rho}$ was arbitrary, we have

$$
\left|u_{e}\right|_{0 ; Q^{\rho}} \leq \frac{N_{2}}{\rho}|u|_{0 ; Q}
$$

Note that by (2.3) with $\frac{\rho}{2}$ in place of $\rho$, we have $\left|u_{e}\right|_{0 ; Q} \frac{\rho}{2} \leq \frac{N_{2}}{\frac{\rho}{2}}|u|_{0 ; Q}$. Now by $(2.2)$ using the domains $Q^{\rho} \subset Q^{\frac{\rho}{2}} \subset Q$ we have

$$
\sup _{Q^{\rho}} u_{e e}^{+} \leq \frac{N_{2}}{\rho}\left|u_{e}\right|_{0 ; Q^{\frac{\rho}{2}}} \leq \frac{N_{2}}{\rho} \cdot \frac{N_{2}}{\frac{\rho}{2}}|u|_{0 ; Q}=\frac{2 N_{2}^{2}}{\rho^{2}}|u|_{0 ; Q}
$$

\section{REFERENCES}

[B] S.N. Bernstein, The Boundedness on the Moduli of a Sequence of Derivatives of Solutions of Equations of Parabolic Type, vol. 18, Dokl. Acad. Nauk SSSR, 1938, pp. 385-388 (Russian).

[Br] A. Brandt, Interior Schauder Estimates for Parabolic Differential (or Difference) Equations via the Maximum Principle, vol. 7, Israel J. Math., 1969, pp. 254-262. MR 40:3044

[C] S. Campanato, Proprietà di una Famiglia di Spazi Functionali, Ann. Scuola Norm. Sup. Pisa (3) 18 (1964), 137-160.

[CC] L. Caffarelli and X. Cabre, Fully Nonlinear Elliptic Equations, Amer. Math. Soc., Providence, R.I., 1995. MR 96h:35046

[E] L.C. Evans, Partial Differential Equations, Amer. Math. Soc., Providence, R.I., 1998. MR 99e:35001

[G] M. Giaquinta, Introduction to Regularity Theory for Nonlinear Elliptic Systems, Birkhäuser Verlag, Basel, 1993. MR 94g:49002

[GT] D. Gilbarg and N. Trudinger, Elliptic Partial Differential Equations of Second Order, 2nd ed., Springer-Verlag, Berlin-Heidelberg-New York-Tokyo, 1983. MR 86c:35035

[K] J. Kovats, Fully Nonlinear Elliptic Equations and the Dini Condition, Communications in PDE 22 (11-12) (1997), 1911-1927. MR 99h:35048]

[K1] N.V. Krylov, Nonlinear Elliptic and Parabolic Equations of the Second Order Equations, Nauka, Moscow, 1987, English transl. Reidel, Dordrecht. MR 88d:35005

[K2] Lectures on Elliptic and Parabolic Equations in Hölder Spaces, Amer. Math. Soc., Providence, R.I., 1996. MR 97i:35001 
[K3] Boundedly Nonhomogeneous Elliptic and Parabolic Equations, vol. 20, Izv. Acad. Nauk., 1983, pp. 459-492, English transl. in Math. USSR Izv.

[KS] N.V. Krylov and M.V. Safonov, Certain Properties of Solutions of Parabolic Equations with Measurable Coefficients, vol. 16, Izv. Acad. Nauk., 1981, pp. 155-164, English transl. in Math. USSR Izv.

[La] E.M. Landis, Second Order Equations of Elliptic and Parabolic Type, vol. 171, Amer. Math. Soc., Providence, R.I., 1998, English transl. in Translations of Math. Monographs. MR 98k:35034

[L] G.M. Lieberman, Intermediate Schauder Theory for Second Order Parabolic Equations IV. Time Irregularity and Regularity, Differential and Integral Equations 5 (1992), 1219-1236. MR 93i:35068

[LSU] O.A. Ladyzhenskaya, V.A. Solonnikov, N.N Ural'tzeva, Linear and Quasilinear Equations of Parabolic Type, vol. 23, Amer. Math. Soc., Providence, R.I., 1968, English transl. in Translations of Math. Monographs.

[M] V.P. Mikhailov, Partial Differential Equations, Mir, Moscow, 1978. MR 82a:35003a

[W] Wang L., On the Regularity Theory of Fully Nonlinear Parabolic Equations: II, Comm. on Pure and Applied Math. 45 (1992), 141-178. MR 92m:35127

Department of Mathematical Sciences, Florida Institute of Technology, Melbourne, FLORIDA 32901

E-mail address: jkovats@zach.fit.edu 